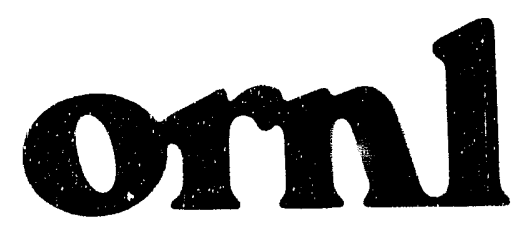

OAK RIDGE NATIONAL. LABORATORY

MARTIN MAGRETRA

\section{Time Optimal Paths for High Speed Maneuvering}

David B. Reister

Suzanne M. Lenhart 
This report has been reproduced directly from the best available copy.

Available to DOE and DOE contractors from the Otfice of Scientific and Techrical Information, P.O. Box 62, Oak Ridge, TN 37831; prices available from (615) 576-8401, FTS 626-8401.

Available to the public from the National Technical Information Service, U.S. Department of Commerce, 5285 Port Royal Rd., Springfield, VA 22161.

This report was prepared as an account of work sponsored by an agency of the United States Government. Neither the United States Government nor any agency thereof, nor any of their employees, makes any warranty, express or implied, or assumes any legal liability or responsibility for the acc'sracy, completeness, or usefulness of any information, apparatus, product, or process disclosed, or represents that its use would not infringe privately owned rights. Reference herein to any specific commercial product, process, or service by trade name, trademark, manufacturer, or otherwise, does not necessarily constitute or imply its endorsement, recommendaticn, or favoring by the United States Government or any agency thereot. The views and opinions of authors expressed herein do not necessarily state or reflect those of the United States Government or any agency thereof. 
Engineering Physics and Mathematics Division

ORNL/TM-12289

TIME OPTIMAL PATHS FOR

DE93 006251 HIGH SPEED MANEUVERING

David B. Reister and Suzanne M. Lenhart

DATE PUBLISHED - January 1993

Research sponsored by the Engineering Research Program Office of Basic Energy Sciences and Office of Nuclear Energy

Office of Technology Support Program

U.S. Department of Energy

Prepared by the

OAK RIDGE NATIONAL LABORATORY

Oak Ridge, Tennessee 37831

managed by

MARTIN MARIETTA ENERGY SYSTEMS, INC.

for the

U.S. DEPARTMENT OF ENERGY

under contract DE-AC05-840R21400 


\section{CONTENTS}

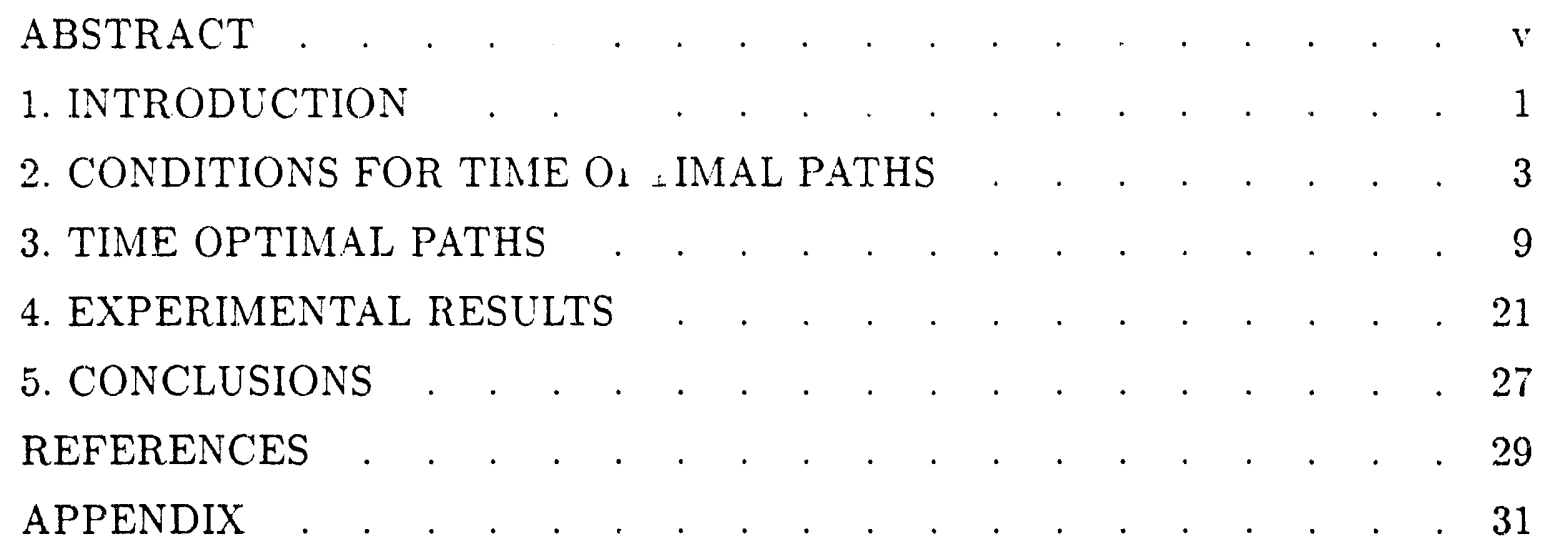




\section{LIST OF FIGURES}

Fig.

1 Time optimal paths when the final orientation is either 0 or 180 degrees

2 Time optimal paths when the final orientation is either 45 or 225 degrees

3 Time optimal paths when the final orientation is either 90 or 270 degrees

4 Time optimal paths when the final orientation is either 135 or 315 degrees

5 Time optimal path to rotate by 180 degrees . . . . . . . . . 15

6 Time optimal paths to reach a position at an arbitrary orientation . 16

7 Lengths of line and circle paths to the point $(2.3,2.0)$ when the final orientation ranges from -210 to 30 degrees . . . . . . . . 17

8 Lengths of line and circle paths to the point $(-2.3,2.0)$ when the final orientation ranges from -30 to 210 degrees . . . . . . . . 18

9 Line and circle paths to the point $(-2.3,2.0)$ when the final orientation is 68 degrees . . . . . . . . . . . . . . 19

10 The target and measured velocity of the right wheel during the experiment. The units of velocity are meters/second . . . . . 22

11 The target and measured orientation of the right wheel during the experiment. The units of orientation are radians

12 The paths of the left and right wheels during the experiment. The units of $x$ and $y$ are meters

13 Calculation of the length of a parallel line path . . . . . . . 33

14 Calculation of the length of a crossover line path . . . . . . 34

15 Calculation of the length of a three circle path . . . . . . . 35 


\begin{abstract}
Recent theoretical results have completely solved the problem of determining the minimum length path for a vehicle with a minimum turning radius moving from an initial configuration to a final configuration. Time optimal paths for a constant speed vehicle are a subset of the minimum length paths. This paper uses the Pontryagin maximum principle to find time optimal paths for a constant speed vehicle. The time optimal paths consist of sequences of arcs of circles and straight lines. The maximum principle introduces concepts (dual variables, bang-bang solutions, singular solutions, and transversality conditions) that provide important insight into the nature of the time optimal paths. We explore the properties of the optimal paths and present some experimental results for a mobile robot following an optimal path.
\end{abstract}




\section{INTRODUCTION}

We consider a mobile robot with one or more steerable drive wheels that steer together (for example, Cybermotion (19'31), Denning (1991), Nomadic (1991), and HERMIES-III (Weisbin, et al. 1990]). We assume that the robot is moving at its maximum speed when it receives orders to drive through a new goal configuration (position and orientation). We want to determine the time optimal path from the current configuration to the goal in an unobstructed environment.

Since the wheels of the mobile robot steer together, we can consider the vehicle to be a unicycle. Time optimal paths for a constant speed vehicle can be minimum length paths. However, there are minimum length paths with cusps that cannot be followed by a constant speed vehicle. Thus, time optimal paths for a constant speed vehicle are a subset of minimum length paths.

Recent theoretical results have completely solved the problem of determining the minimum length path for a vehicle moving from an initial configuration to a final configuration. In general, the optimal paths consist of sequences of arcs and lines. In 1957, Dubins proved that the minimal length paths without cusps (with Lipschitz continuous tangents) are either arc-line-arc (ALA) or arc-arc-arc (AAA). In 1990, Reeds and Shepp determined the minimum length paths with cusps.

Sussmann and Tang (1991) have derived the results of Reeds and Shepp using geometric methods based on the Lie algebraic analysis of trajectories. Boissonnat and his colleagues (1992) have derived the results of Reeds and Shepp using the Pontryagin Maximum Principle (Pontryagin et al. 1962). Soueres and Laumond (1992) have synthesized paths using the results of Reeds and Shepp.

Several recent papers (Jacobs and Canny 1989; Pin and Vasseur 1990; and Vasseur, et al. 1991) have explored minimum length paths for mobile robots that have a minimum turning radius. These authors have developed path planning algorithms for complex environments containing obstacles.

In this paper, we will use the Pontryagin Maximum Principle to find time optimal paths for a constant speed vehicle that has one degree of freedom (the steering angle). Our results were obtained independently of the work by Boissonnat (1992). We will find that the optimal paths are either bang-bang or singular. Furthermore, the bang-bang paths are arcs and the singular paths are lines. Thus, the optimal paths consist of sequences of arcs and lines. Using the result of Dubins (1957), the optimal paths are either arc-line-arc (ALA) or arc-arc-arc (AAA). The maximum principle introduces concepts (dual variables, bang-bang solutions, singular solutions, and transversality conditions) that provide important insight into the nature of the minimum time paths. We will not consider path planning algorithms for complex environments containing obstacles.

The next section will use the maximum principle and Dubin's results to derive the conditions for time optimal paths. The third section will explore the features of the optimal paths. The fourth section presents some experimental results for a mobile robot following an optimal path, while the fifth section lists our conclusions. 


\section{CONDITIONS FOR TIME OPTIMAL PATHS}

The basic equations of motion for a single wheel are:

$$
\begin{aligned}
& \dot{x}=v \cos \phi \\
& \dot{y}=v \sin \phi
\end{aligned}
$$

where the Cartesian coordinates $(x, y)$ locate the point of contact between the wheel and the floor, $\dot{x}$ is the $x$ component of the wheel velocity $(v)$, and $\phi$ is the orientation of the plane of the wheel with respect to the $x$ axis. We assume that the velocity of the wheel orientation is the control variable:

$$
\dot{\phi}=u
$$

where the magnitude of the orientation velocity is bounded: $|u| \leq a$. While the control input could be the acceleration of the wheel orientation, the velocity is the control variable that we use for the HERMIES-III vehicle (Reister 1992).

To apply the Pontryagin Maximum Principle, we introduce three state variables: $x=(x, y, \phi)$. The three state variables define the configuration (position and orientation) of the vehicle. In vector notation, the equations of motion for the state vector $x$ are:

$$
\dot{x}=f(x, u)
$$

The components of the equations of motion are:

$$
\begin{gathered}
\dot{x}_{1}=f_{1}(x, u)=v \cos x_{3} \\
\dot{x}_{2}=f_{2}(x, u)=v \sin x_{3} \\
\dot{x}_{3}=f_{3}(x, u)=u
\end{gathered}
$$

The optimization problem is to find a path for the control variable $[u]$ that will move the system from the initial configuration $x^{0}$ to the final configuration $x^{1}$ and minimize the transition time.

Pontryagin introduces a system of dual variables $[\Psi]$ that satisfy:

$$
\dot{\Psi}_{i}=-\sum_{j=1}^{3} \frac{\partial f_{j}(x, u)}{\partial x_{i}} \Psi j, \quad i=1,2,3 .
$$


The equations of motion for the dual variables are:

$$
\begin{gathered}
\dot{\Psi}_{1}=0 \\
\dot{\Psi}_{2}=0 \\
\dot{\Psi}_{3}=\Psi_{1} v \sin x_{3}-\Psi_{2} v \cos x_{3}
\end{gathered}
$$

The initial conditions are:

$$
\Psi_{i}\left(t_{0}\right)=\mu_{i} \quad i=1,2,3 .
$$

If $x_{3}$ is known, Eqs. (9) to (11) are linear and homogeneous and have a unique solution for any initial conditions.

Pontryagin combines the equations of motion and the dual variables into a single Hamiltonian, $H$ :

$$
\begin{gathered}
H(\Psi, x, u)=\sum_{j=1}^{3} \Psi_{j} f_{j}(x, u) \\
H(\Psi, x, u)=\Psi_{1} f_{1}(x)+\Psi_{2} f_{2}(x)+\Psi_{3} u
\end{gathered}
$$

Pontryagin proves that the optimal set of control variables maximizes the Hamiltonian. Since the Hamiltonian is linear in the control variable $u$, the optimal solution is bang-bang or singular. The sign of the third dual variable $\left(\Psi_{3}\right)$ determines the sign of the optimal control. When $\Psi_{3}$ is positive (negative), the optimal control is bang-bang and at its upper (lower) limit. When $\Psi_{3}$ is zero for an interval, the optimal control is singular and may have intermediate values.

We will show that the optimal path consists of a sequence of arcs and line segments by proving the following Theorem.

Theorem 1. The optimal control can only be bang-bang or singular. When the control is bang-bang, the control is at its limit and the path is an arc of a circle. When the control is singular, the control is zero on a finite interval and the path is a line segment.

We consider first the case where the control is singular. Thus, $\Psi_{3}=0$ on a finite interval. Using Eqs. (5) and (6), Eq. (11) may be written:

$$
\dot{\Psi}_{3}=\Psi_{1} \dot{x}_{2}-\Psi_{2} \dot{x}_{1}
$$

To simplify our notation, we can perform a space-time coordinate transformation. We will assume that the initial point on the time interval is zero and we will choose the coordinate systern to make the initial values of the state variables equal $(0,0,0)$. In the new coordinate system, Eq. (15) can be integrated to yield:

$$
\Psi_{3}=\mu_{1} y-\mu_{2} x+\mu_{3}
$$


Since $\Psi_{1}$ and $\Psi_{2}$ are constants, we have replaced them with their initial values $\left(\mu_{1}\right.$ and $\mu_{2}$ ).

When the control is singular, $\Psi_{3}$ is zero and Eq. (16) defines a line. On the line, $\phi$ is a constant and $\dot{\phi}=0$. Thus, $u=0$ on the finite interval where $\Phi=0$.

A line can be defined by the inner product of a position vector $(r)$ and a vector (b) that is perpendicular to the line:

$$
r \cdot b=c
$$

The constant (c) is proportional to the distance from the line to the origin (see Section 1.4 of Paul 1981). For Eq. (16), the vectors are: $r=(x, y)$ and $b=\left(-\mu_{2}, \mu_{1}\right)$. Thus, $\Psi_{3}=r \cdot b+\mu_{3}$. The parameter $\left(\mu_{3}\right)$ is proportional to the distance from the line where $\Psi_{3}$ is zero to the origin (when $\Psi_{3}=0, \mu_{3}=-c$ ) and the value of the dual variable $\left(\Psi_{3}\right)$ is proportional to the distance from the point $(x, y)$ to the line where $\Psi_{3}$ is zero. In the next section, we will find that the line determines the optimum solution. Typically, the optimal control steers the wheel to the line, follows the line, and then steers to the goal.

We consider next the case where the control is bang-bang. We will consider a finite interval where $\Psi_{3}$ is positive (or negative) and $u$ is a constant. We shall show that the path is an arc of a circle on the finite interval. As in the singular case, we will assume that the initial point on the time interval is zero and we will choose the coordinate system to make the initial values of the state variables equal $(0,0,0)$. When the control is constant initially, Eq. (3) can be integrated to yield: $\phi=u t$. Equations (1) and (2) can be integrated to find the Cartesian path:

$$
\begin{gathered}
x=(v / u) \sin \phi \\
y=(v / u)(1-\cos \phi)
\end{gathered}
$$

Thus, the path is an arc of a circle. The radius of the circle is the ratio of the wheel velocity and the steering velocity $[R=|(v / u)|]$. The curvature of the circle is the reciprocal of the radius. The center of the circle is $(0, q): x^{2}+(y-q)^{2}=R^{2}$, where $q=v / u(R=|q|)$.

We have proven Theorem 1. The optimum path consists of a sequence of arcs and line segments. Next we establish upper limits on the number of arcs and line segments.

While the control variable $(u)$ can be discontinuous, the orientation $(\phi)$ will be continuous. Thus, the components of the wheel velocity ( $\dot{x}$ and $\dot{y})$ will be continuous. Thus, at all points, the paths will have continuous tangents. At any point on a line segment, the control variable could become positive or negative and the path would become an arc of a circle. At the transition point from line to circle, the line must be tangent to the circle. Similarly, at any point on a circular path, the control variable could change sign and the path would switch to an arc of another circle. At the transition point from circle to circle, the two circles must be tangent. The 
dual variable $\Psi_{3}$ is continuous. At the transition point from circle to circle, $\Psi_{3}=0$ and the transition point from circle to circle must lie on the line $\Psi_{3}=0$.

If a path consists of a line segment $\left(\Psi_{3}=0\right)$ followed by a circle, the circle is tangent to the line. Hence the path on the circle cannot cross the line and the path cannot leave the circle and get back on the line $\left(\Psi_{3}=0\right)$ except at the original point of departure. Thus no minimum time path can consist of two distinct segments of the line $\left(\Psi_{3}=0\right)$ joined by an arc of circle. The arc must be a full circle and the segments can not be distinct. No minimum time path can contain a full circle.

We can imagine a long sequence of tangent circles that have all of the points of tangency on a single line. As we were attempting to bound the number of arcs in an optimum path, the paper by Jacobs and Canny (1989) led us to the seminal results of Dubins (1957).

Before stating Dubins' theorem, we need to specify some notation. Dubins considers curves with "average curvature always less than or equal to R-1," which means that for each curve the tangent vector exists everywhere and satisfies the Lipschitz condition:

$$
\left\|\mathrm{X}^{-1}\left(s_{1}\right)-X^{-1}\left(s_{2}\right)\right\| \leq R^{-1}\left|s_{1}-s_{2}\right|
$$

where $X$ is a vector with components $(x, y)$ and $s$ is the path length. A portion of a path that is an arc of a circle with radius $R$ is denoted by $A$. A line segment is denoted by $L$. Since the tangents are continuous, the lines and circles are tangent to each other. Dubins has proven the following theorem.

Theorem 2. Given an initial position and orientation in the plane $(P)$ and a final position and orientation $(Q)$. The minimum length path from $P$ to $Q$ that has an average curvature everywhere less than or equal to $R^{-1}$ is necessarily a continuously differentiable curve that is either ALA; or AAA; or a subpath of a path of type ALA or AAA.

Our set of candidates for optimal paths consist of sequences of arcs and line segments. Dubins considers a more general class: curves with average curvature always less than or equal to $R^{-1}$. Since the minimum length paths for the more general class are in our class, the minimum length paths for our class are the same as Dubins'. Hence, we conclude that our optimal paths are either ALA; or AAA; or subpaths.

We have assumed that all three state variables are specified at the end of the path. We could be interested in paths with sorne free boundary conditions. We might want to reach a point $(x, y)$ at an arbitrary orientation or we might want to reach an orientation at an arbitrary point. When an optimal path has free boundary conditions, Pontryagin's Maximum Principle determines the optimal solution. We assume that the goal $x^{1}$ is a point in a smooth manifold $S$. Let $T$ be the tangent plane to $S$ at the goal. The dual solution satisfies the transversality condition if it is orthogonal to $T$. Pontryagin's Maximum Principle requires that the dual solution must satisfy the transversality condition at the goal. If the goal is to reach a point at an arbitrary orientation, the tangent plane is defined by the vector $(0,0,1)$ and the transversality condition requires that $\Psi_{3}=0$ at the goal. Thus, the last 
segment of the path to the goal is a line. If the goal is to reach an orientation at an arbitrary point, the tangent plane is defined by the vector $(1,1,0)$ and the transversality condition requires that $\Psi_{1}=\Psi_{2}=0$ at the goal. Thus, $\Psi_{3}$ is a constant and the path to the goal is an arc. 


\section{TIME OPTIMAL PATHS}

We have defined the features of time optimal paths. In this section, we will explore time optimal paths for several classes of problems. We begin by considering the case where both position and orientation are specified at the goal. We will choose the coordinate system to make the initial values of the state variables equal $(0,0,0)$. The time optimal paths will be ALA or AAA or subpaths. Unless the time optimal paths are subpaths, the initial and final segments of the optimum path will be arcs. Thus, given an initial state $(0,0,0, \ldots 1$ a final state $(x, y, \phi)$, we can draw the two circles that lead away from the initial state and the two circles that lead into the final state. Next we look for lines or circles that will connect an initial circle to a final circle.

Figures 1 to 4 illustrate optimal paths to a point for eight different orientations. In the figures, the initial and final circles are drawn with dotted lines while the optimal paths are drawn with solid lines. The initial state is always the same and the initial pair of circles is always the same. As the orientation of the final state changes, the final pair of circles rotate. In Fig. 1, the paths lead from the initial poin $\$(S)$ to the fina: point $(G)$ and the orientation is either 0 or 180 degrees. The path to 0 degrees (path $b$ ) starts with a positive (counterclockwise) arc and ends with a negative (clockwise) arc. The path to 180 degrees (path $a$ ) starts and ends with a positive arc. In Fig. 2, the orientation is either 45 or 225 degrees. The path to 45 degrees (path $b$ ) ends in a line segment. In Fig. 3, the orientation is either 90 or 270 degrees. Compared to Fig. 1, the concluding arcs have switched circles. The path to 90 degrees (path $a$ ) starts and ends with a positive arc. The path to 270 degrees (path $b$ ) starts with a positive arc and ends with a negative arc. In Fig. 4, the orientation is either 135 or 315 degrees.

For the paths in Figs. 1 to 4, we could choose a consistent set of values for the initial conditions of the dual variables and verify that the paths are optimal. The parameters $\left(\mu_{1}, \mu_{2}\right)$ define the slope of the line segment, we will define $\mu_{1}$ and $\mu_{2}$ by: $\mu_{1}=\cos \theta$ and $\mu_{2}=\sin \theta$, where $\theta$ is the slope of the line segment. The distance from $(0,0)$ to the line and the sign of the optimal control determine $\mu_{3}$. For example in Fig. 4, the path to 135 degrees (path $a$ ) starts and ends with a positive control. Thus, $\mu_{3}$ is the positive distance from $(0,0)$ to the line. Both the initial point and the goal are on the left side of the line. In Fig. 4, the path to 315 degrees (path $b$ ) starts with a positive control and ends with a negative control. The initial point is on the left side of the line while the goal is on the right side of the line.

A constant speed vehicle cannot make tight maneuvers. Figure 5 shows the path required to turn around [the goal is $(0,0,180)]$. The path consists of three arcs. For this case, the line $\left(\Psi_{3}=0\right)$ is vertical; the optimal contrul is negative on the left of the line and positive on the right (or vice versa the path could be traveled in either direction).

While time optimal paths at constant velocity are similar to minimum length paths, they have one significant difference. A minimum length path can reverse direction at a point (have a cusp). Our minimum time paths cannot have 
discontinuities in tangent vectors. If a vehicle needs to perform tight maneuvers, it should not move at constant speed. It should slow down. As the speed $(v)$ approaches zero, the turning radius $(R)$ approaches zero (recall that $R=|(v / u)|$ ).

If the goal is to reach a position at an arbitrary orientation, the last segment of the path to the goal is a line. Paths that can reach a point are explored in Fig. 6. Figure 6 displays the paths when the steering velocity is positive. A similar set of paths could be obtained if the steering velocity is negative [the point $(x, y)$ is mapped to $(x,-y)$ ]. For goals outside the two circles defined by the radius of curvature, we have truncated the lengths of the line segments to remain within the region where the paths are optimal [in the upper half plane $(y>0)$ ]. The paths displayed in Fig. 6 are optimal to reach any point in the upper half plane except the points inside the circle. Furthermore, they are optimal to reach any point within the circle for the lower half plane. The paths with negative steering velocity are optimal for the complementary regions (inside the upper circle and outside the lower circle).

We have explored the optimal paths to a point for eight different orientations. Next we will discuss an algorithm that will find the optimal path from an initial state to a final state. The time optimal paths will be ALA or AAA. The initial and final segments of the optimum path will be arcs. Since we have two initial circles and two final circles, we have four combinations of an initial circle and a final circle. Given an initial circle and a final circle for the ALA case, we define two types of line paths that join the two circles: parallel and crossover. The parallel paths are parallel to the line that joins the centers of the two circles while the crossover path cuts the line that joins the two circles. In Fig. 1, path a is a parallel path and path $b$ is a crossover path. Although we can always find a parallel path between two circles, we cannot find a crossover path between two intersecting circles (if the distance between the centers of the two circles is less than $2 R$ ). In the appendix, we derive expressions for the lengths of parallel and crossover paths.

Given an initial circle and a final circle for the AAA case, we define two types of circle paths that join the two circles: left and right. The center of the left circle is to the left of the line joining the centers of the two circles while the center of the right circle is to the right of the line. In the appendix, we derive expressions for the lengths of circle paths. Circle paths will not exist if the distance between the centers of the two circles is more than $4 \mathrm{R}$.

To find the optimal path from an initial state to a final state, we have four combinations of an initial circle and a final circle. For each combination of an initial circle and a final circle, we can have a maximum of four paths (two line paths and two circle paths). Thus, we can have a maximum of 16 paths from the initial state to the final position. Half of the paths will have the correct orientation and half of the paths will have the opposite orientation. Thus, to find the optimum path, we calculate the lengths of the 8 paths with the proper orientation and choose the shortest path. 


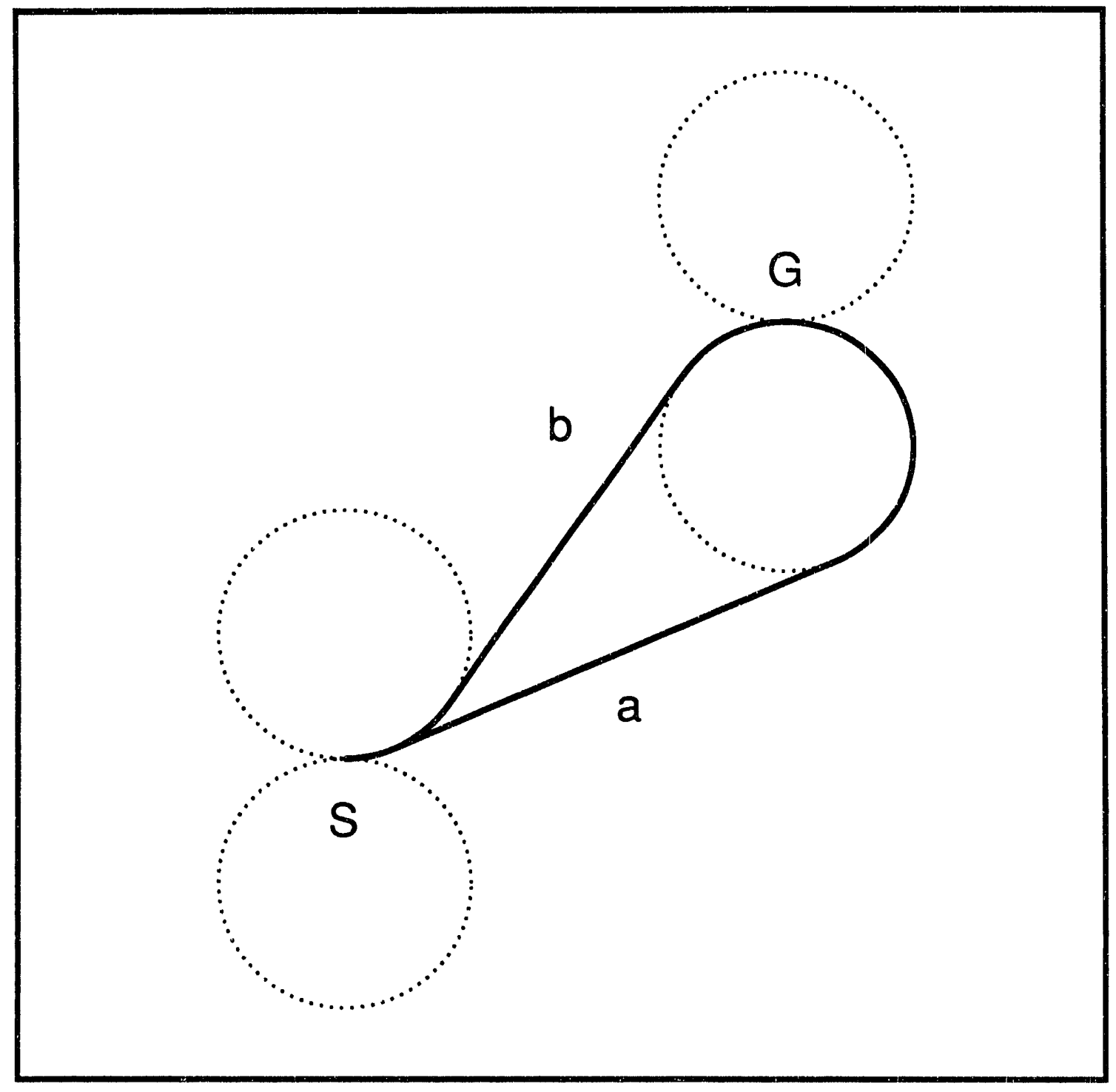

Fig. 1. Time optimal paths when the final orientation is either 0 or 180 degrees. 


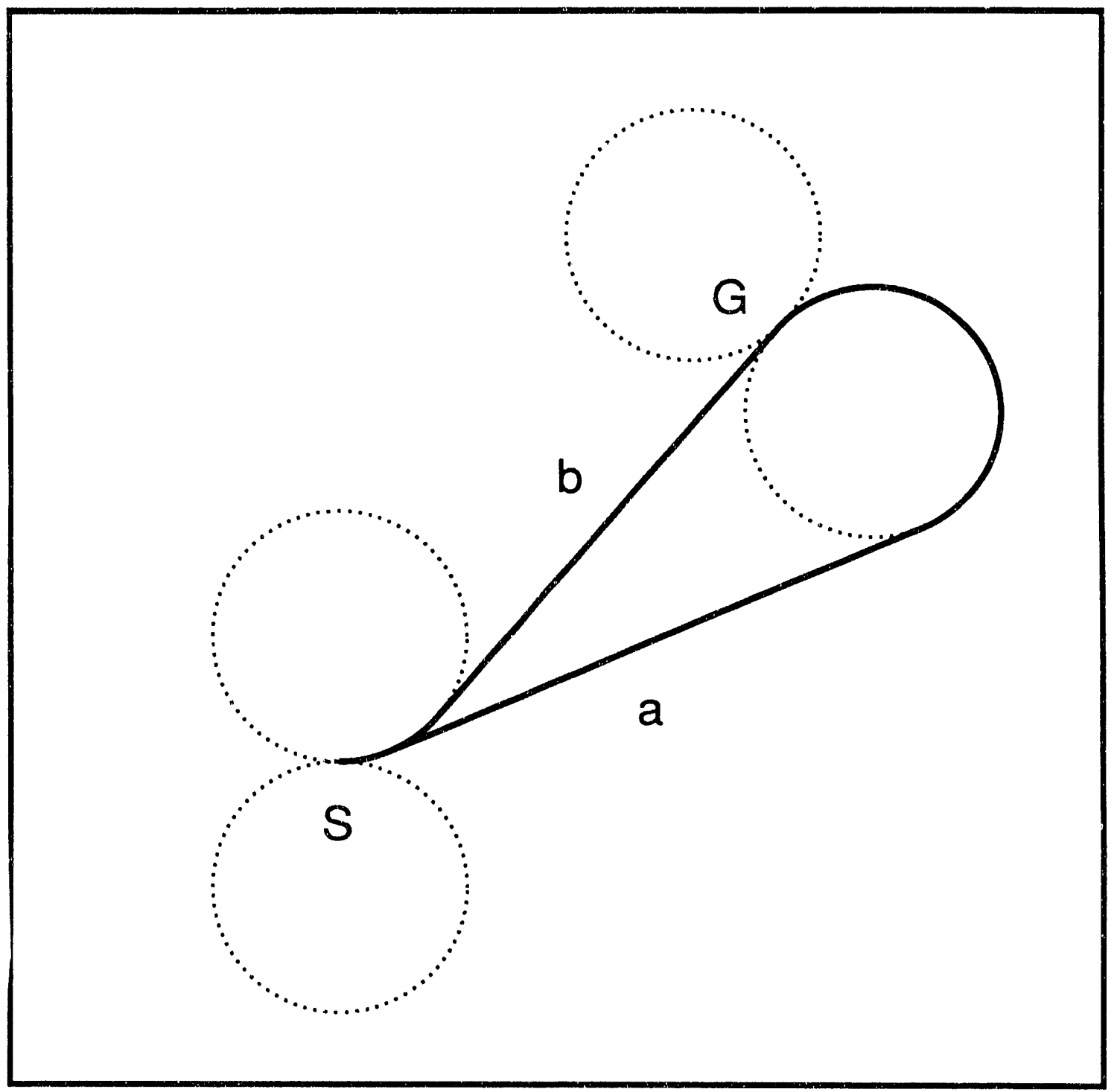

Fig. 2. Time optimal paths when the final orientation is either 45 or 225 degrees. 


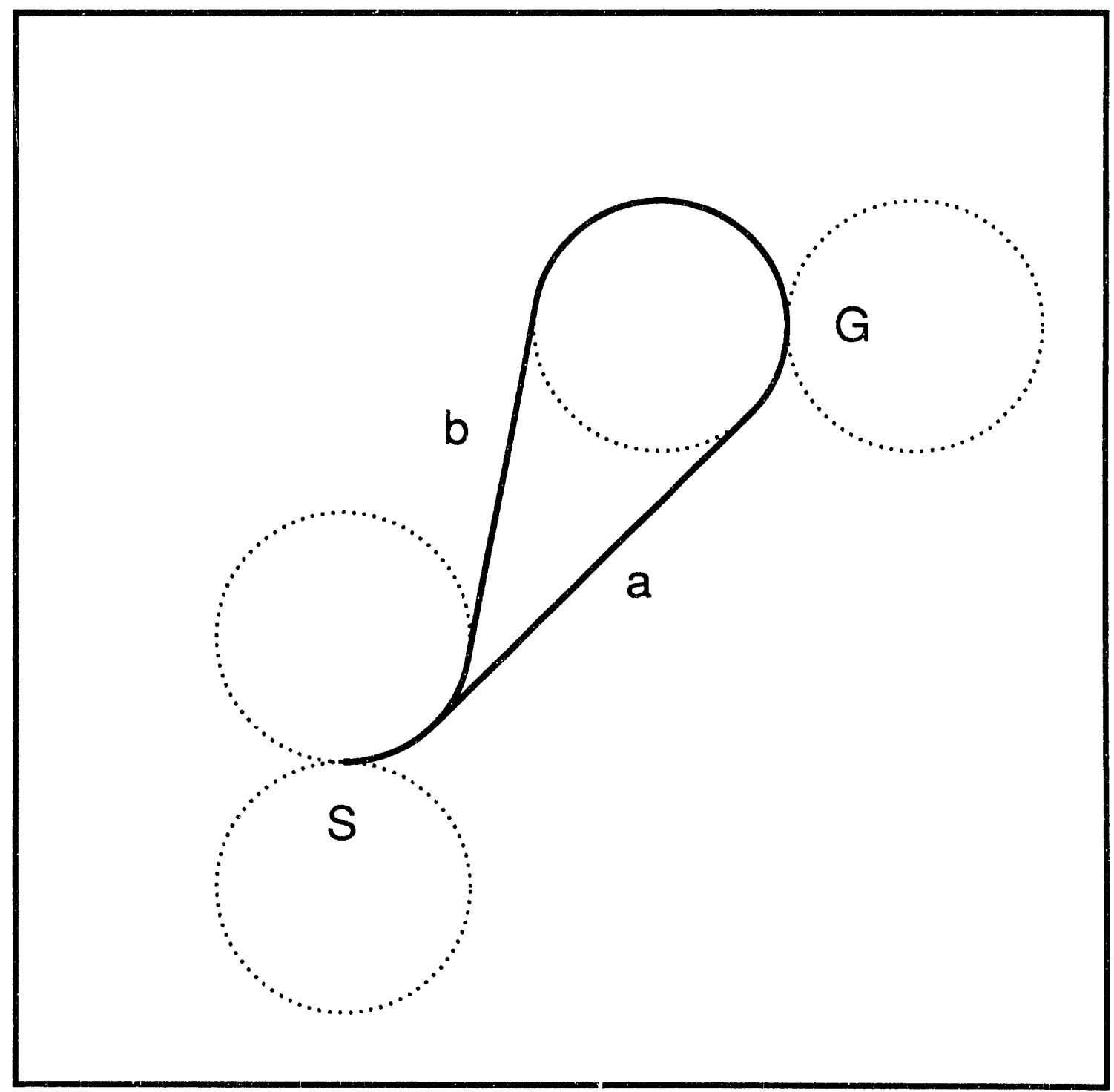

Fig. 3. Time optimal paths when the final orientation is either 90 or 270 degrees. 
14 TIME OPTIMAL PATHS

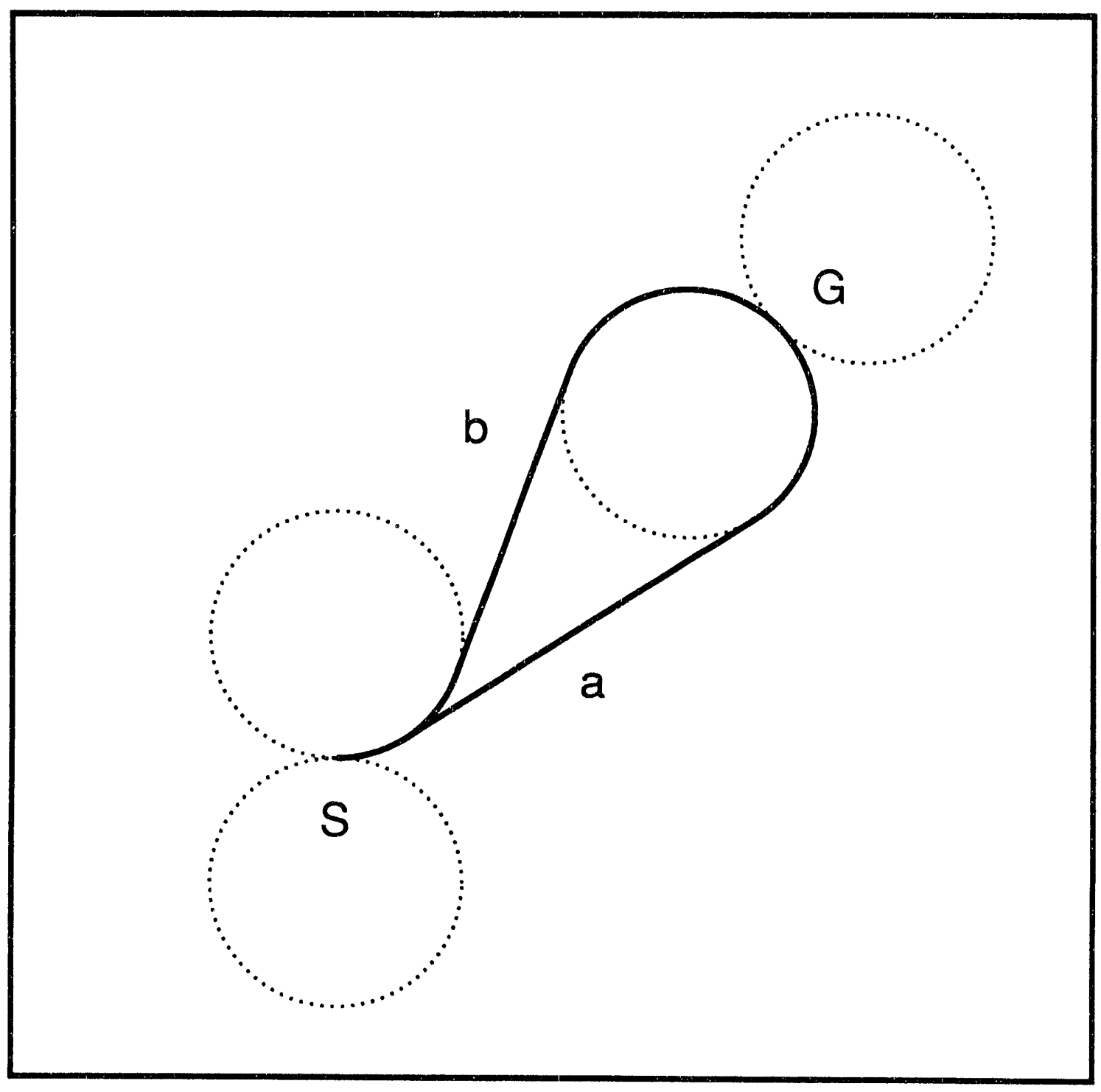

Fig. 4. Time optimal paths when the final orientation is either 135 or 315 degrees. 


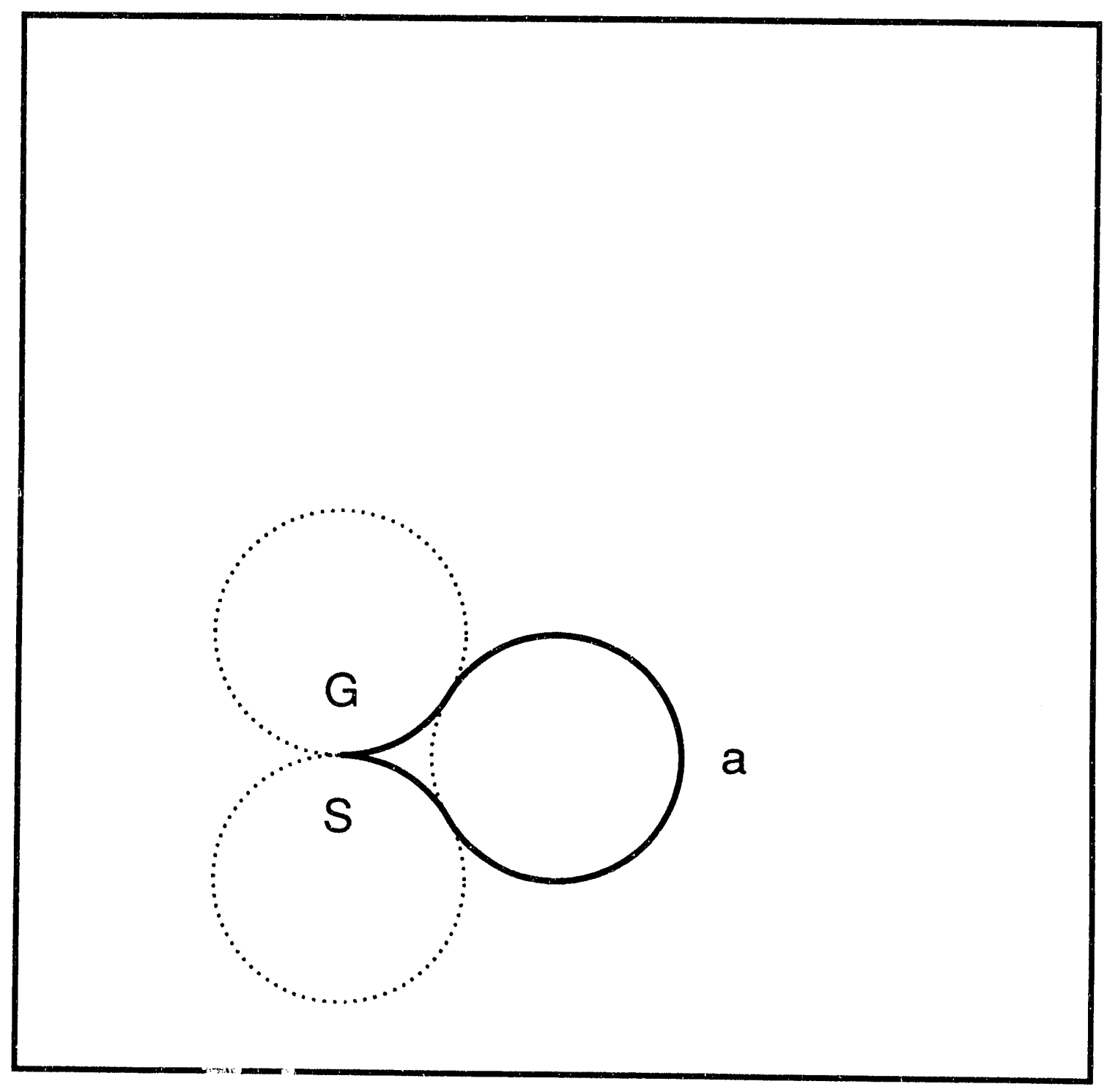

Fig. 5. Time optimal path to rotate by 180 degrees. 


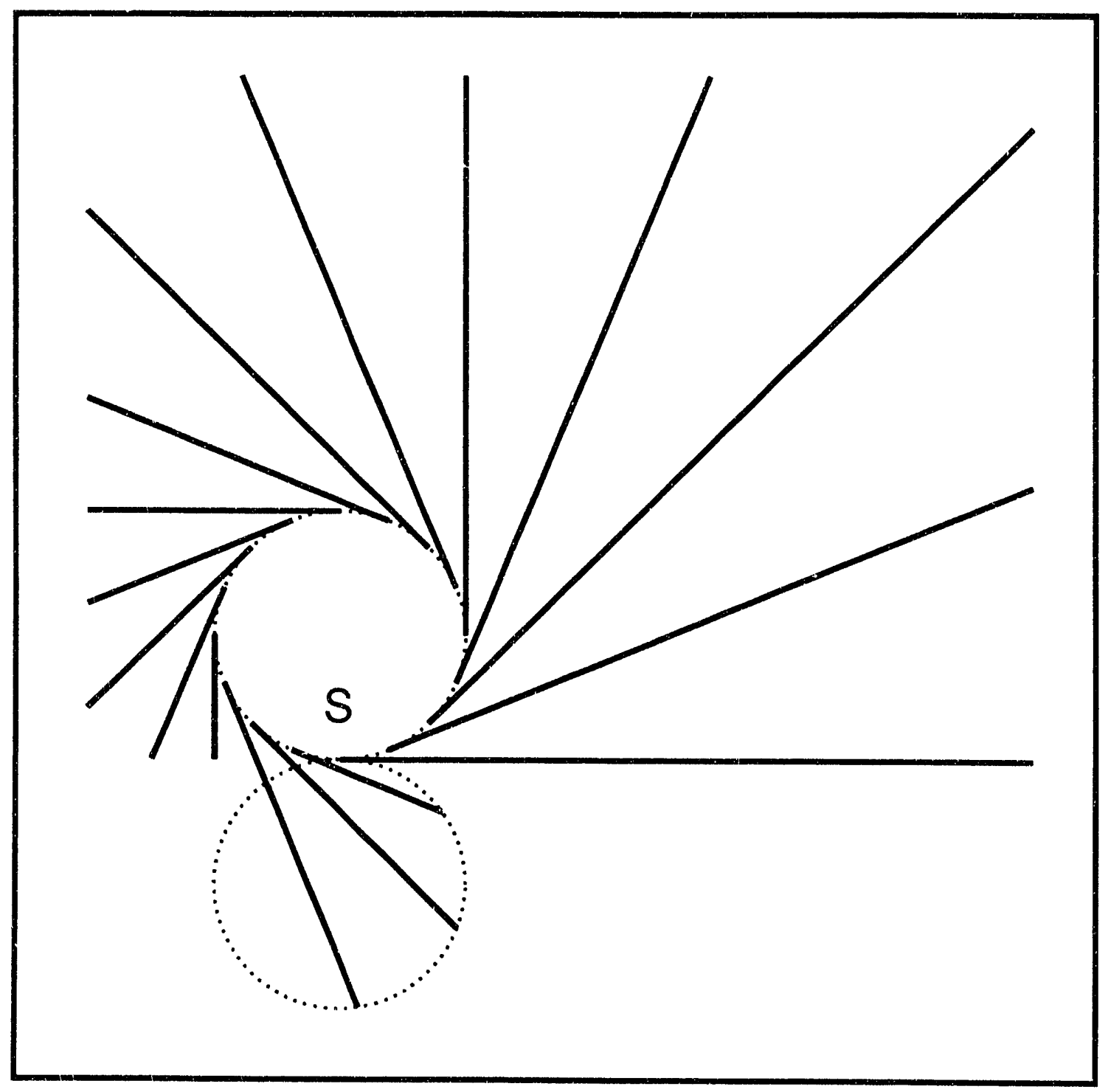

Fig. 6. Time optimal paths to reach a position at an arbitrary orientation.

The lengths of the shortest line and circle paths from the initial state $(0,0,0)$ to the firal state $(2.3,2.0, \phi)$ are displayed in Fig. 7. The line paths are the optimal paths for all final orientations except the interval from -114 degrees to -18 degrees. The lengths of the shortest line and circle paths to the final state $(-2.3,2.0, \phi)$ are displayed in Fig. 8. The line paths are the optimal paths for all final orientations except the interval from 68 degrees to 114 degrees. 


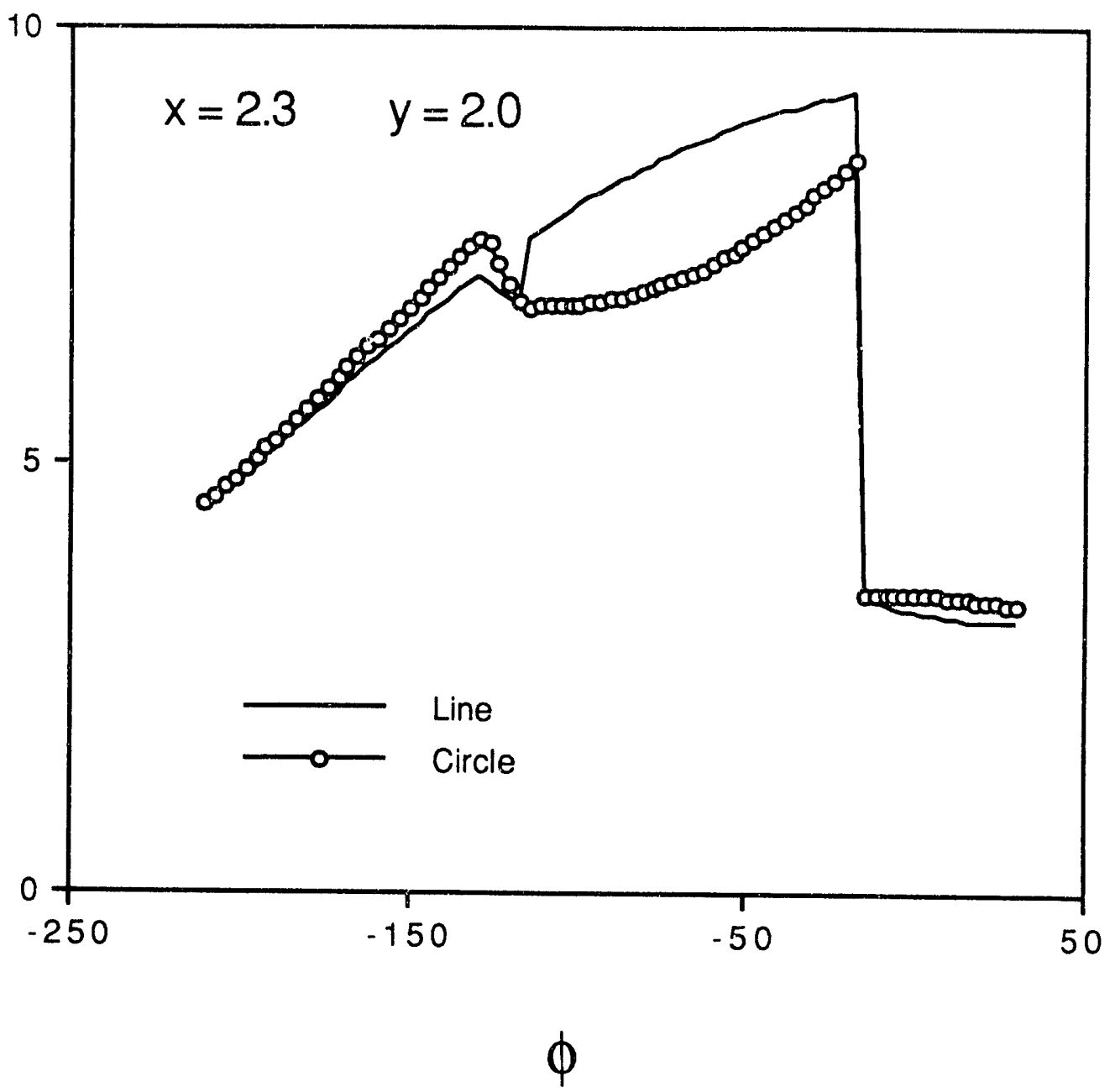

Fig. 7. Lengths of line and circle paths to the point $(2.3,2.0)$ when the final orientation ranges from -210 to 30 degrees.

We now explore further the transition from ALA to AAA in Fig. 8. The shortest line and circle paths to the final state $(-2.3,2.0,68$ degrees $)$ are displayed in Fig. 9. At 68 degrees, both paths have the same length. As the orientation increases from 68 degrees to 114 degrees, the final pair of circles rotates about the point $G$ and the circle path $b$ is the optimal path. After 114 degrees, the circles are not intersecting and the crossover path becomes the shortest path.

Dubins proves that when the optimal path is AAA, the angle subtended by the middle arc must be greater than 180 degrees. We have examined several numerical examples where the optimal path is AAA and we have verified that the angle subtended by the middle arc of each path is greater than 180 degrees. In Fig. 9, the angle subtended by the middle arc of the AAA path $(b)$ is greater than 180 degrees. 


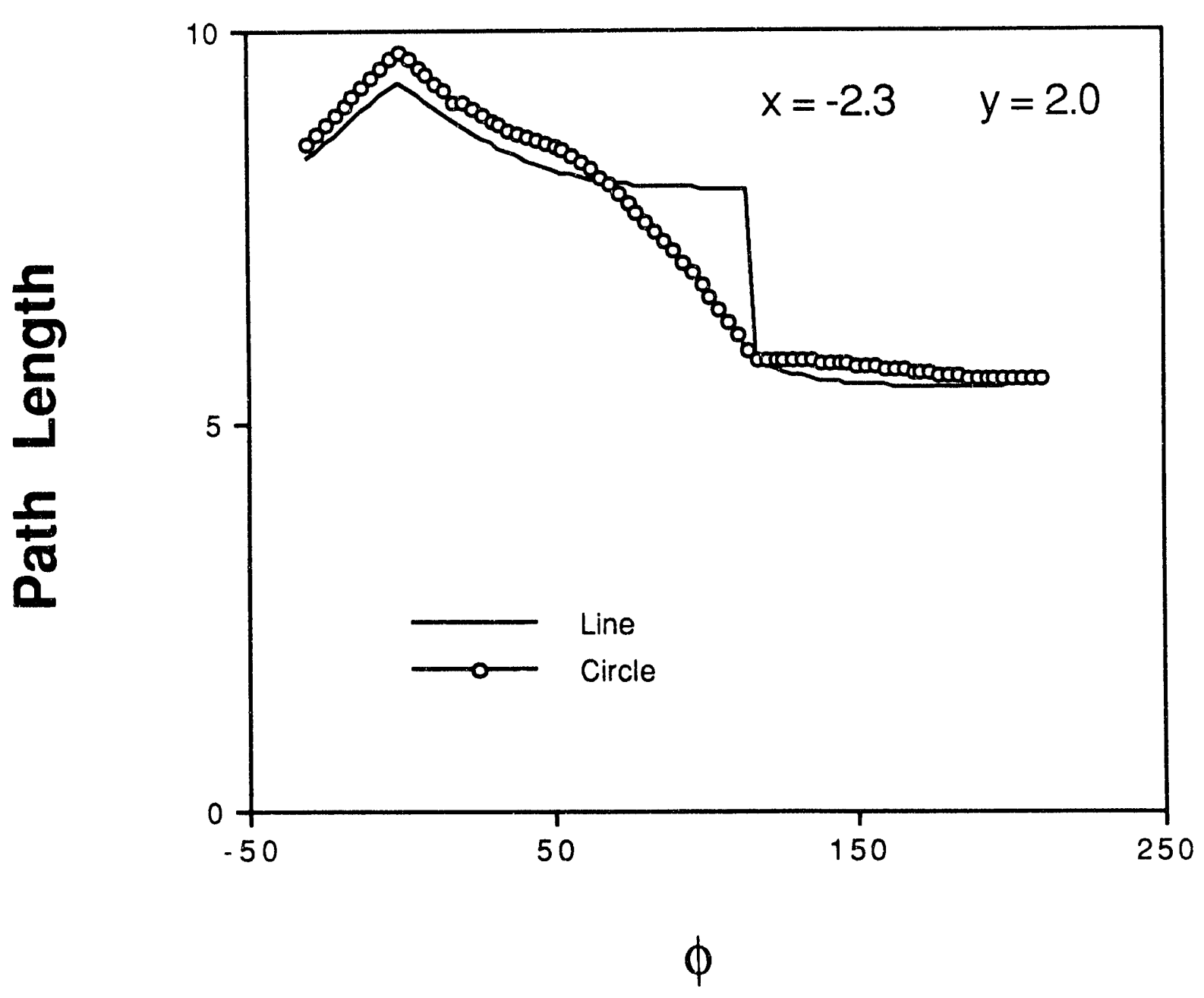

Fig. 8. Lengths of line and circle paths to the point $(-2.3,2.0)$ when the final orientation ranges from $\mathbf{- 3 0}$ to 210 degrees. 


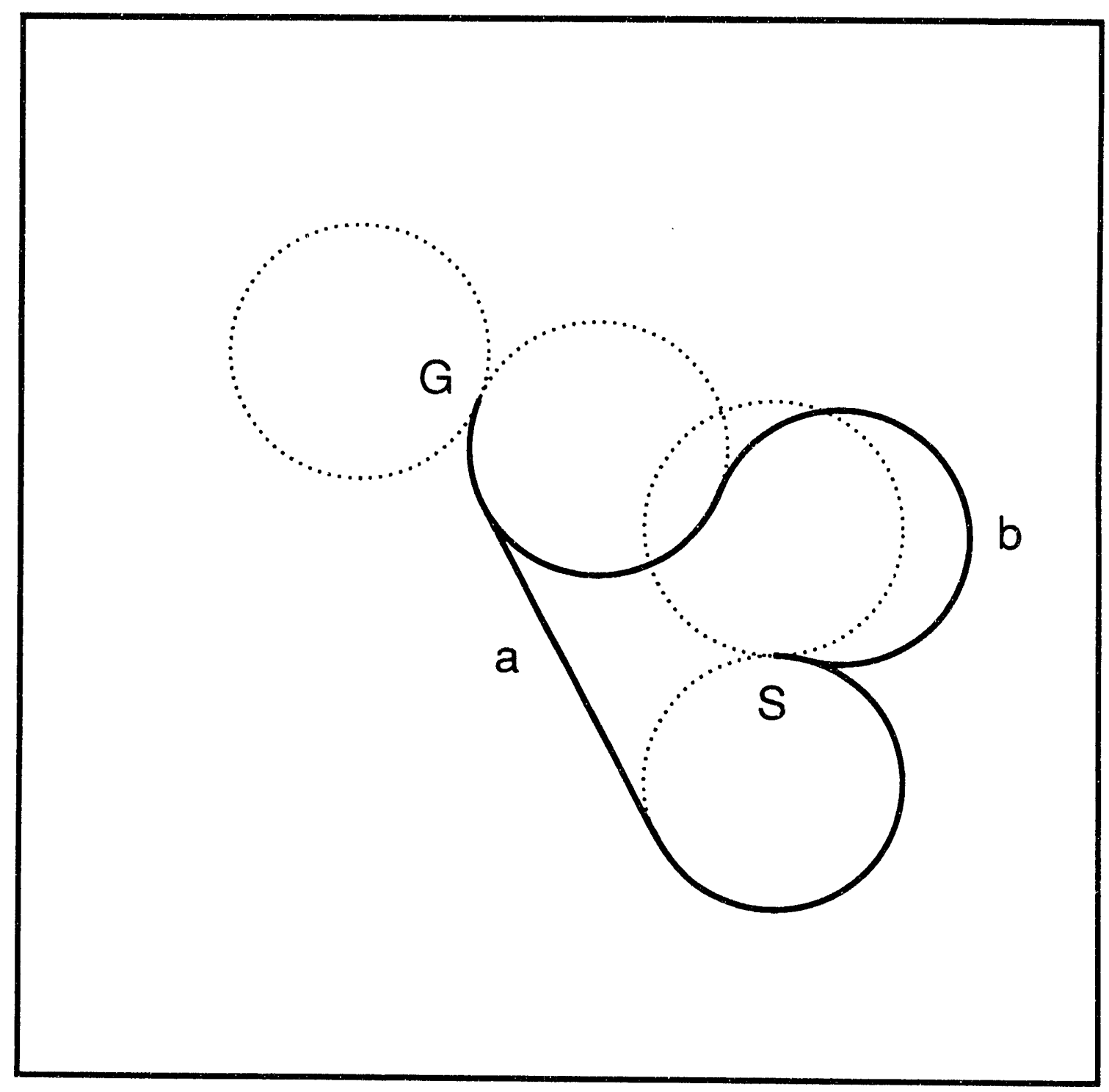

Fig. 9. Line and circle paths to the point $(-2.3,2.0)$ when the final orientation is 68 degrees. 


\section{EXPERIMENTAL RESULTS}

The objective of our experiment is to demonstrate that a mobile robot can follow an ALA type path. Our mobile robot is HERMIES-III, a large robot designed for human scale experiments (Reister 1992). The chassis $(1.6 \mathrm{~m} \times 1.3 \mathrm{~m} \times 1.9 \mathrm{~m})$ has two steerable drive wheels and four corner caster wheels. The vehicle weighs $820 \mathrm{~kg}$. The maximum wheel velocity is $0.45 \mathrm{~m} / \mathrm{s}$ and the maximum velocity for wheel orientation is 60 degrees/second (1.05 radians/second). The radius of the arc is the ratio of the wheel velocity and the steering velocity $[R=|(v / u)|]$ [see Eqs. (18) and (19)]. Thus, at maximum speed for the vehicle and for steering, the turning radius is 0.43 meters.

During the experiment, the vehicle accelerates to full speed, steers left on an arc for 0.75 seconds to an orientation of 45 degrees, follows a 45 degree line for one secord, steers right for 45 degrees, and decelerates to a stop. During each cycle of the wheel control system (approximately 33 times per second), the robot saves key data elements that are downloaded from memory to disk after the experiment.

Using data collected during an experiment, the target and measured values for the wheel speed are displayed in Fig. 10. At 7.64 seconds, the target for the wheel speed was raised from 0.0 to $0.45 \mathrm{~m} / \mathrm{s}$. By 9.86 seconds, the vehicle was at full speed and the vehicle remained at full speed until the target was reduced to 0.0 at 12.91 seconds. By 15.04 seconds, the vehicle had stopped.

The target and measured values for the wheel orientation are displayed in Fig. 11. At 10.15 seconds, the target for the wheel orientation was raised from 0.0 to 0.79 radians ( 45 degrees). By 11.02 seconds, the wheel orientation was at 45 degrees and the orientation remained at 45 degrees until the target was reduced to 0.0 at 11.90 seconds. By 12.71 seconds, the measured orientation was 0.0 . Since the vehicle was at full speed from 9.86 seconds to 12.91 seconds, it was at full speed during the period ( 10.15 to 12.71 seconds) when the wheels were being steered and the vehicle was following the path.

The paths for the two steerable drive wheels are displayed in Fig. 12. The midpoint between the two wheels starts at the point $(4.00,6.00)$ and ends at the point $(6.10,6.61)$. During the experiment, the vehicle accelerates to full speed while moving in the $x$ direction, steers left on an arc to an orientation of 45 degrees, follows a 45 degree line, steers right for 45 degrees, and decelerates to a stop while moving in the $x$ direction. The net motion is 2.10 meters in $x$ and 0.61 meters in $y$.

At the start of the high speed maneuver (10.15 seconds), the midpoint between the two wheels is at the point $(4.62,6.01)$. At the end of the maneuver (12.71 seconds), the midpoint is at the point $(5.54,6.60)$. Thus the net motion during the maneuver is 0.92 meters in $x$ and 0.59 meters in $y$ in 2.56 seconds. The line connecting the start and end of the maneuver is 1.09 meters and the average velocity $(1.09 / 2.56=0.43 \mathrm{~m} / \mathrm{s})$ is close to the target for wheel speed $(0.45 \mathrm{~m} / \mathrm{s})$. 


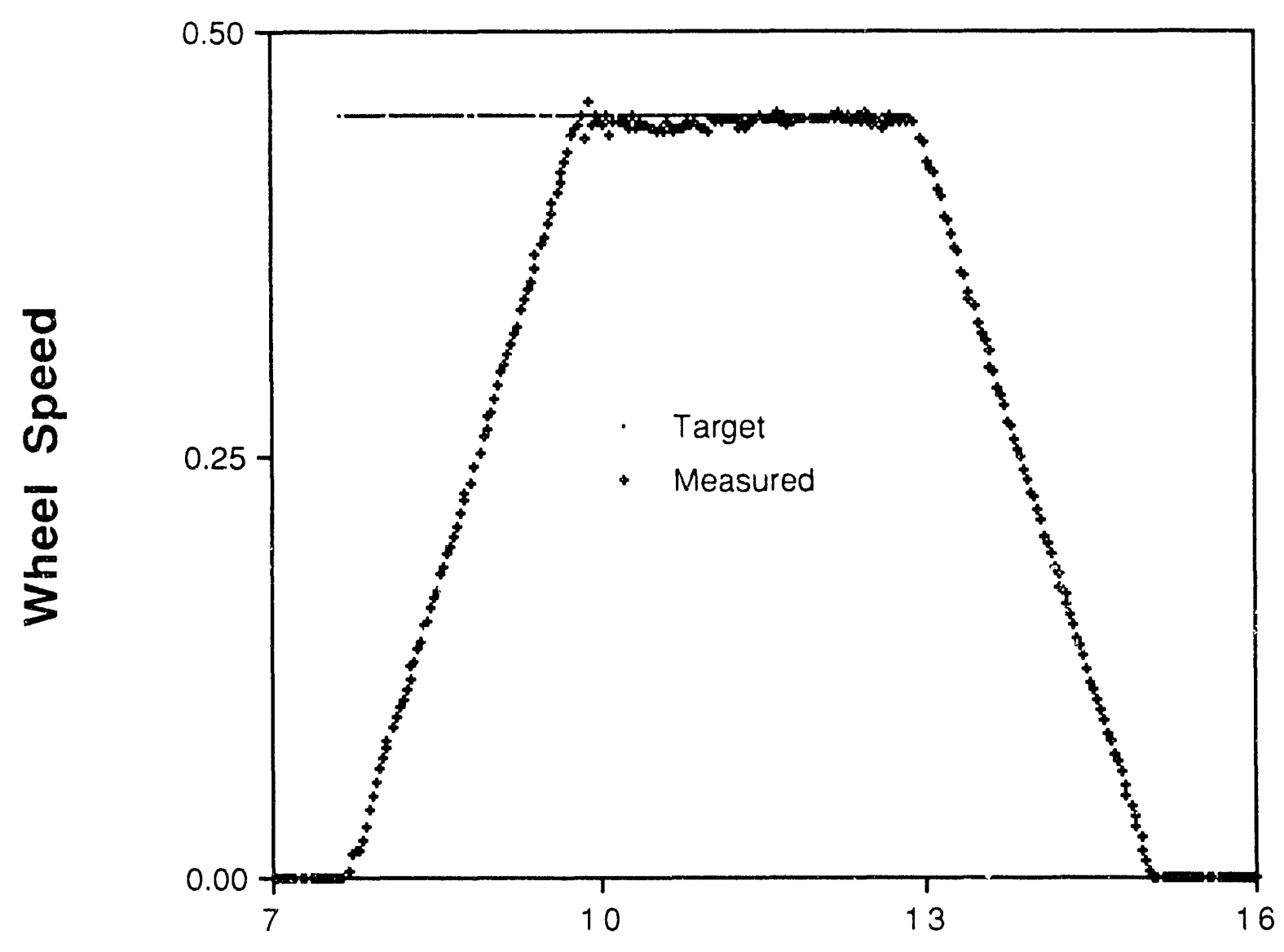

Time

Fig. 10. The target and measured velocity of the right wheel during the experiment. The units of velocity are meters/second.

This experiment marks the first time that a mobile robot has followed a minimum time ALA type path. While the figures illustrate that the vehicle did not follow the path exactly, the errors were small. We will conclude this section by quantifying the errors. 


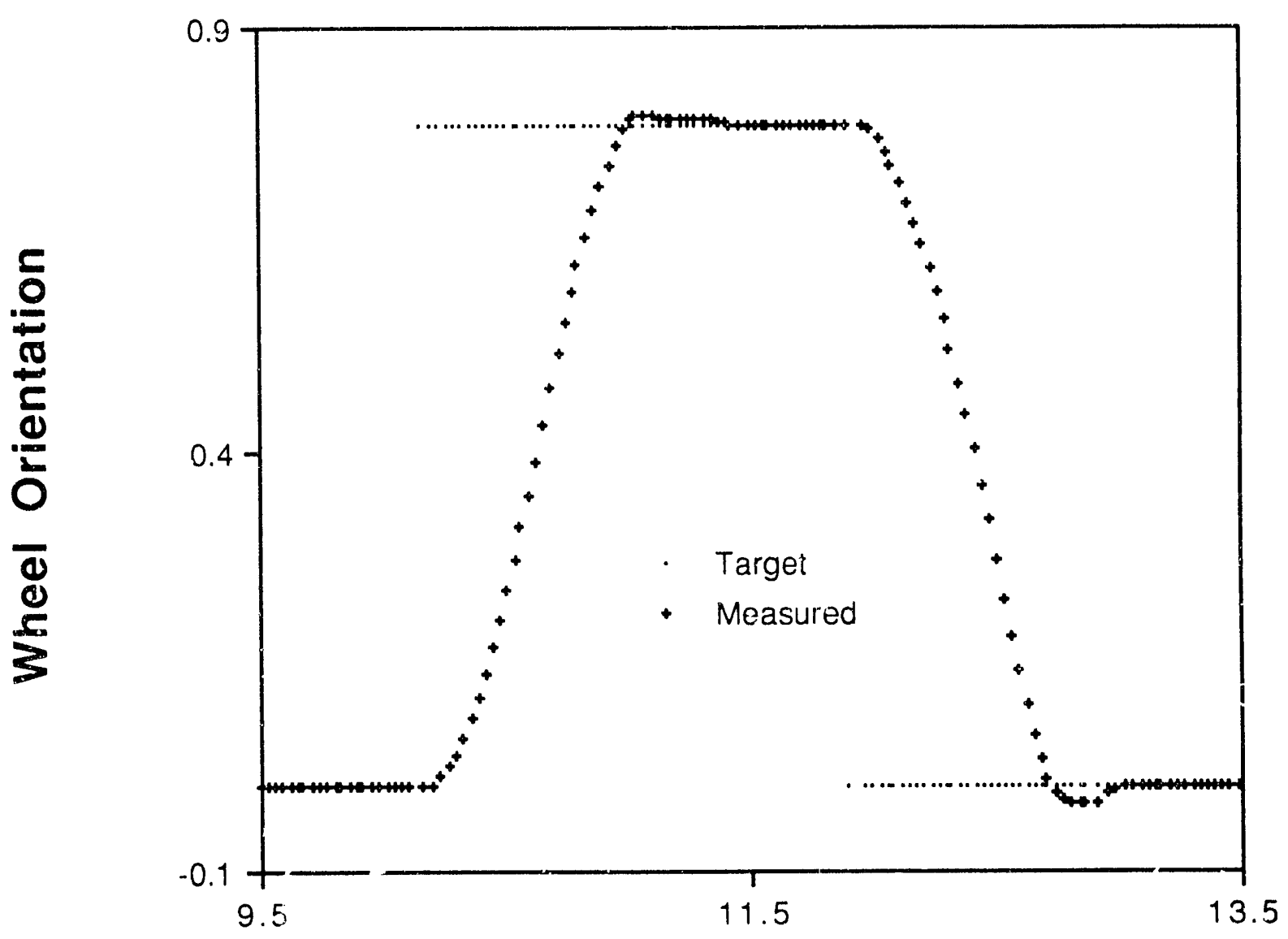

\section{Time}

Fig. 11. The target and measured orientation of the right wheel during the experiment. The units of orientation are radians.

To quantify the errors in Fig. 10, we fitted the best (least squares linear regression) line to the velocity data for the period when the vehicle was at full speed (from 9.91 seconds to 12.91 seconds). The best fit line has a small slope. During the period ( 10.15 to 12.71 seconds) when the vehicle was following the path, the linear fit to the velocity increased from $0.445 \mathrm{~m} / \mathrm{s}$ to $0.448 \mathrm{~m} / \mathrm{s}$. The largest errors were $-1.2 \%$ at 10.69 seconds and $1.1 \%$ at 11.65 seconds. Thus, most of the measured velocities are within $1.0 \%$ of the least squares line through the data. 


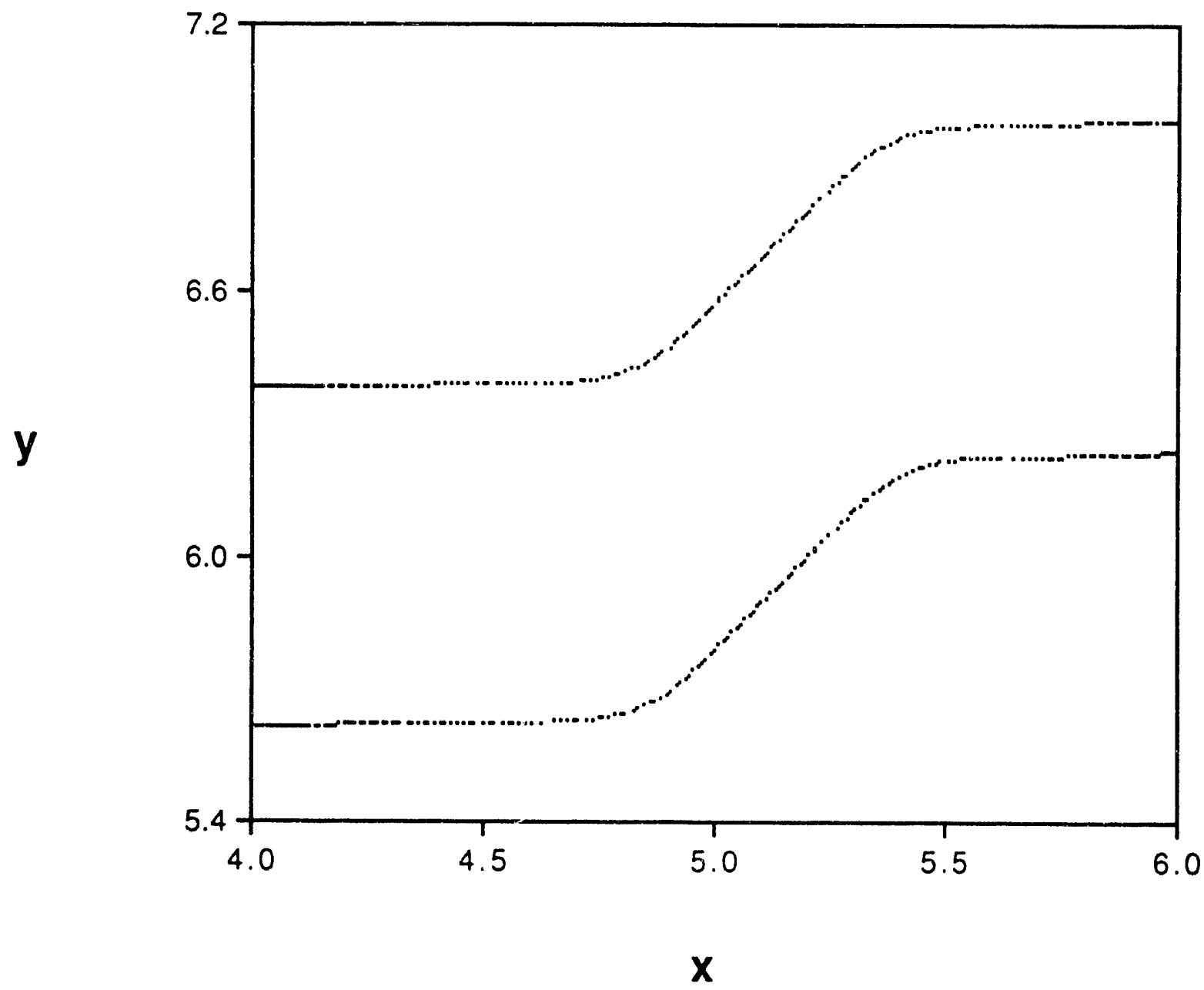

Fig. 12. The paths of the left and right wheels during the experiment. The units of $x$ and $y$ are meters.

To quantify the errors in Fig. 11, we divided the data into three groups (Arc, Line, and Arc). Using linear regression, we fitted the best line to the data during the first period (when the wheel orientation increases from 0 degrees to 45 degrees). As the well known statistical parameter $R^{2}$ increases from 0.0 to 1.0 , the parameter measures how well the linear model explains the variation in the data. During the first period, the vaiue of $R^{2}$ was 0.989 . During the third period, the wheel orientation decreases from 45 degrees to 0 degrees and the value of $R^{2}$ was 0.981 . Thus, in both cases the measured data is very close fit to a linear model. 
During the second period, the target for the wheel orientation is 45 degrees. Initially, the measured data for the wheel orientation overshoots the target before approaching the target. The maximum value for the overshoot is $1.4 \%$.

Thus, we have demonstrated that the errors between the targets and the measured values for both the velocity and the orientation were small. 


\section{CONCLUSIONS}

We desire time optimal paths for a mobile robot (with one or more steerable drive wheels that steer together) that is traveling at its maximum speed. Recent theoretical results have completely determined the nature of the time optimal paths. The optimal paths consist of sequences of arcs and lines and are either ALA or AAA. In this paper, we have used the Pontryagin Maximum Principle to find time optimal paths for a constant speed vehicle. The time optimal paths are produced by control trajectories that are either bang-bang or singular. The bang-bang controls lead to subpaths that are arcs of circles, while the singular controls produce line segments.

When the final configuration is not fully specified, the transversality condition determines the optimal solution. To reach a point at an arbitrary orientation, the path is AL (an initial arc followed by a line segment). To reach an orientation at an arbitrary point, the whole path is an arc.

We have developed an algorithm that will find the optimal path from an initial state to a final state. Since we have two initial circles and two final circles, we have four combinations of an initial circle and a final circle. Given an initial circle and a final circle, we define two types of line paths that join the two circles: parallel and crossover and two types of circle paths that join the two circles: left and right. For each combination of an initial circle and a final circle, we can have a maximum of four paths (two line paths and two circle paths). Thus, we can have a maximum of 16 paths from the initial state to the final position. Half of the paths will have the correct orientation and half of the paths will have the opposite orientation. Thus, to find the optimum path, we calculate the lengths of the 8 paths with the proper orientation and choose the shortest path.

Using HERMIES-III, we have demonstrated that a large mobile robot can follow an ALA type path with small errors.

For practical applications, the paths are best for high speed maneuvering in unobstructed environments and are not appropriate for maneuvering in complex environments or for large changes in orientation. For example, the rotation path in Fig. 5 required the vehicle to turn 420 degrees $(+60,-300,+60)$ to accomplish a 180 degree change in orientation. This maneuver should be performed when the vehicle is at rest. 


\section{REFERENCES}

1. J. D. Boissonnat, A. Cerezo, and J. Leblond, "Shortest Paths of Bounded Curvaturein the Plane," Proc. IEEE International Conference on Robotics and Automation, 2315-2320 (1992).

2. Cybermotion. Roanoke, Virginia.

3. Denning Mobile Robotics, Inc., Wilmington, Massachusetts.

4. L. E. Dubins, "On Curves of Minimal Length with a Constraint on Average Curvature, and with Prescribed Initial and Terminal Positions and Tangents," American Journal of Mathematics 79, 497-516 (1957).

5. P. Jacobs and J. Canny, "Planning Smooth Paths for Mobile Robots," Proc. IEEE International Conference on Robotics and Automation, 2-7 (1989).

6. Nomatic Technologies, Inc., Palo Alto, California.

7. R. P. Paul, Robot Manipulators: Mathematics, Programming, and Control, MIT Press, Cambridge, Massachusetts.

8. F. G. Pin and H. A. Vasseur, "Autonomous Trajectory Generation for Mobile Robots with Non-Holonomic and Steering Angle Constraints," Proc. IEEE International Workshop on Intelligent Motion Control, 295-299 (1990).

9. L. S. Pontryagin et al., "The Mathematical Theory of Optimal Processes" John Wiley, New York (1962).

10. J. A. Reeds and L. A. Shepp, "Optimal Paths for a Car that Goes Both Forwards and Backwards," Pacific Journal of Mathematics 145 367-393, (1990).

11. D. B. Reister, "A New Wheel Control System for the Omnidirectional HERMIES-III Robot, Robotica 10, 351-360 (1992).

12. P. Soueres and J. P. Laumond, "Synthese des Plus Courts Chemins pour la Voiture de Reeds et Shepp," LAAS/CNRS 92234, Laboratory d'Automatique et d'Analyse des Systems, Toulouse, France (1992).

13. H. J. Sussmann and G. Tang, "Shortest Paths for the Reeds-Shepp Car: A Worked Out Example of the Use of Geometric Techniques in Nonlinear Optimal Control," SYCON-91-10, Rutgers University, New Brunswick, New Jersey.

14. H. A. Vasseur, F. G. Pin, and J. R. Taylor,"Navigation of a Car-Like Mobile Robot Using a Decomposition of the Environment in Convex Cells, Proc. IEEE International Conference on Robotics and Automation," 1496-1502 (1991).

15. C. R. Weisbin et al., "HERMIES-III: A Step Toward Autonomous Mobility, Manipulation, and Perception," Robotica 8, 7-12 (1990). 


\section{APPENDIX}

\section{CALCULATION OF PATH LENGTHS}

The optimal path is either arc-line-arc or arc-arc-arc. To find the optimal path, we calculate the lengths of all possible arc-line-arc or arc-arc-arc paths and pick the shortest path. In this appendix, we will derive formulas for the path lengths. We begin with the arc-line-arc paths.

We associate two circles with both the initial position and the final position. Given one of the initial circles and one of the final circles, we can define two types of connecting lines: parallel and crossover. A parallel line is parallel to the line that joins the centers of the two circles while the crossover path cuts the line that joins the centers of the two circles.

The calculation of the length of a parallel line path is diagrammed in Fig. 13. The path consists of an arc of length $\alpha R$, followed by a line of length $d$, followed by an arc of length $\beta R$. The path length $(L)$ is:

$$
L=\alpha R+d+\beta R
$$

where $R$ is the radius of the circles.

To derive expressions for $\alpha, d$, and $\beta$, we introduce the following notation: $r$ is the distance from the center of the initial circle to the center of the final circle, $\theta$ is the orientation of the line that joins the centers of the two circles, $\sigma$ is the orientation of the line from the center of the initial circle to the starting point $(S)$, $\rho$ is the orientation of the line from the center of the final circle to the goal point $(G)$, and $\lambda$ is the orientation of the line from the center of the final circle to the end of the line segment (or the end of the middle arc).

Using our notation, we can derive expressions for $\alpha, d$, and $\beta$.

$$
\begin{gathered}
\alpha=\theta \\
d=r \\
\lambda=\sigma+\theta \\
\beta=\rho-\lambda
\end{gathered}
$$




\section{Appendix}

The calculation of the length of a crossover line path is diagramed in Fig. 14. The path consists of an arc of length $\alpha R$, followed by a line of length $d$, followed by an arc of length $\beta R$. The path length $(L)$ is given by Eq. (20). The angle between the crossover path and the line that joins the centers of the two circles is $\gamma$ :

$$
\gamma=\arcsin (2 R / r)
$$

When $r$ is less than $2 R$, the two circles intersect and the crossover path is not possible. Using our previous notation, we can derive expressions for $\alpha, d$, and $\beta$.

$$
\begin{gathered}
\alpha=\theta+\gamma \\
d=r \cos \gamma \\
\lambda=\alpha+\pi / 2 \\
\beta=\lambda-\rho
\end{gathered}
$$

The calculation of the length of a three circle path is diagramed in Fig. 15. The path consists of an arc of length $\alpha R$, followed by an arc of length $\beta R$, followed by an arc of length $\gamma R$. The path length $(L)$ is:

$$
L=R(\alpha+\beta+\gamma)
$$

The angle between the line that joins the centers of the first two circles and the line that joins the centers of the initial and final circles is $\delta$ :

$$
\delta=\arccos (r / 4 R)
$$

When $r$ is greater than $4 R$, the middle circle cannot bridge from the initial circle to the final circle. Using our previous notation, we can derive expressions for $\alpha, \beta$, and $\gamma$.

$$
\begin{gathered}
\alpha=\theta-\delta-\sigma \\
\beta=\pi-2 \delta \\
\lambda=\theta+\delta-\pi \\
\gamma=\rho-\lambda
\end{gathered}
$$




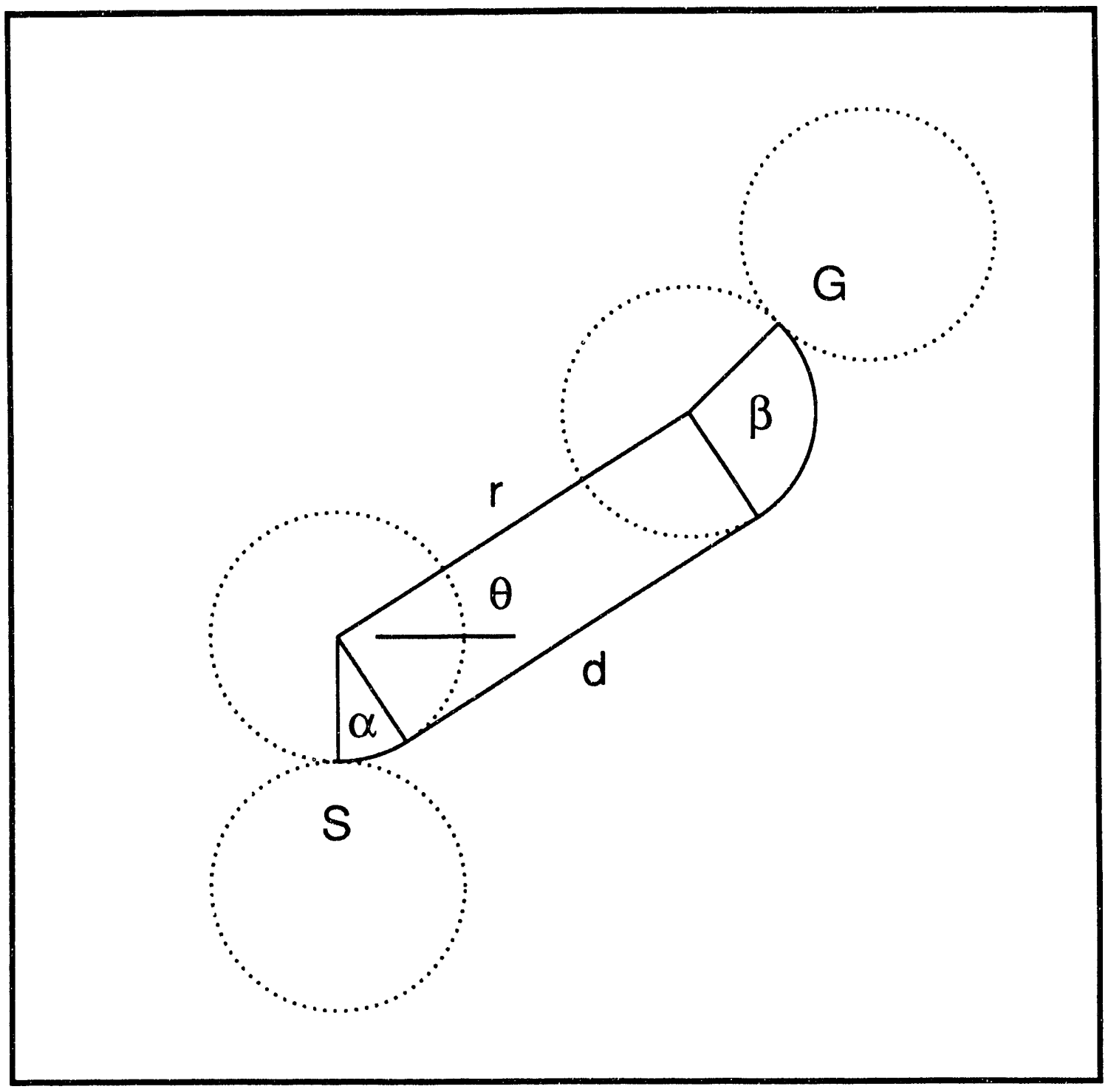

Fig. 13. Calculation of the length of a parallel line path. 


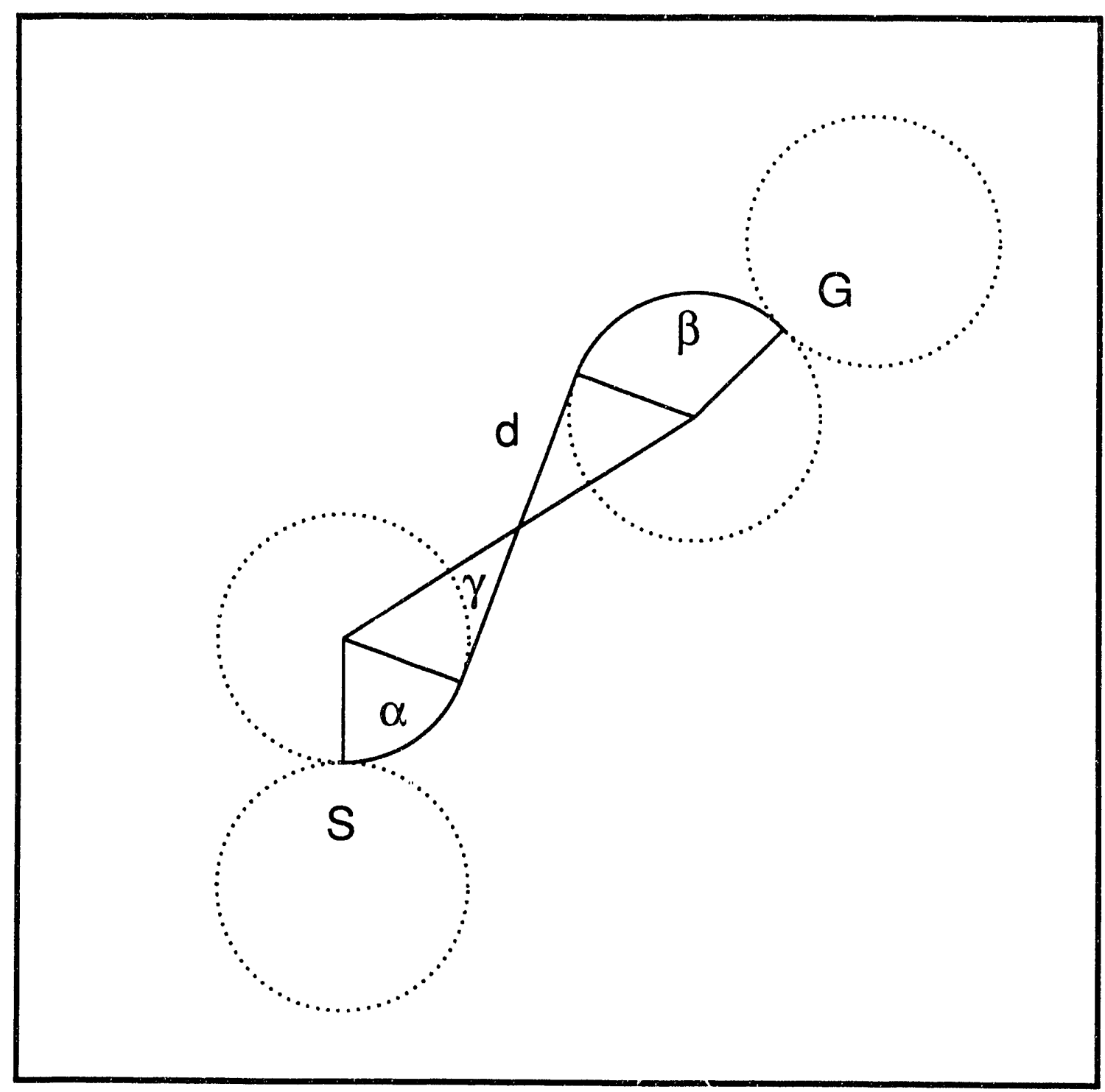

Fig. 14. Calculation of the length of a crossover line path. 


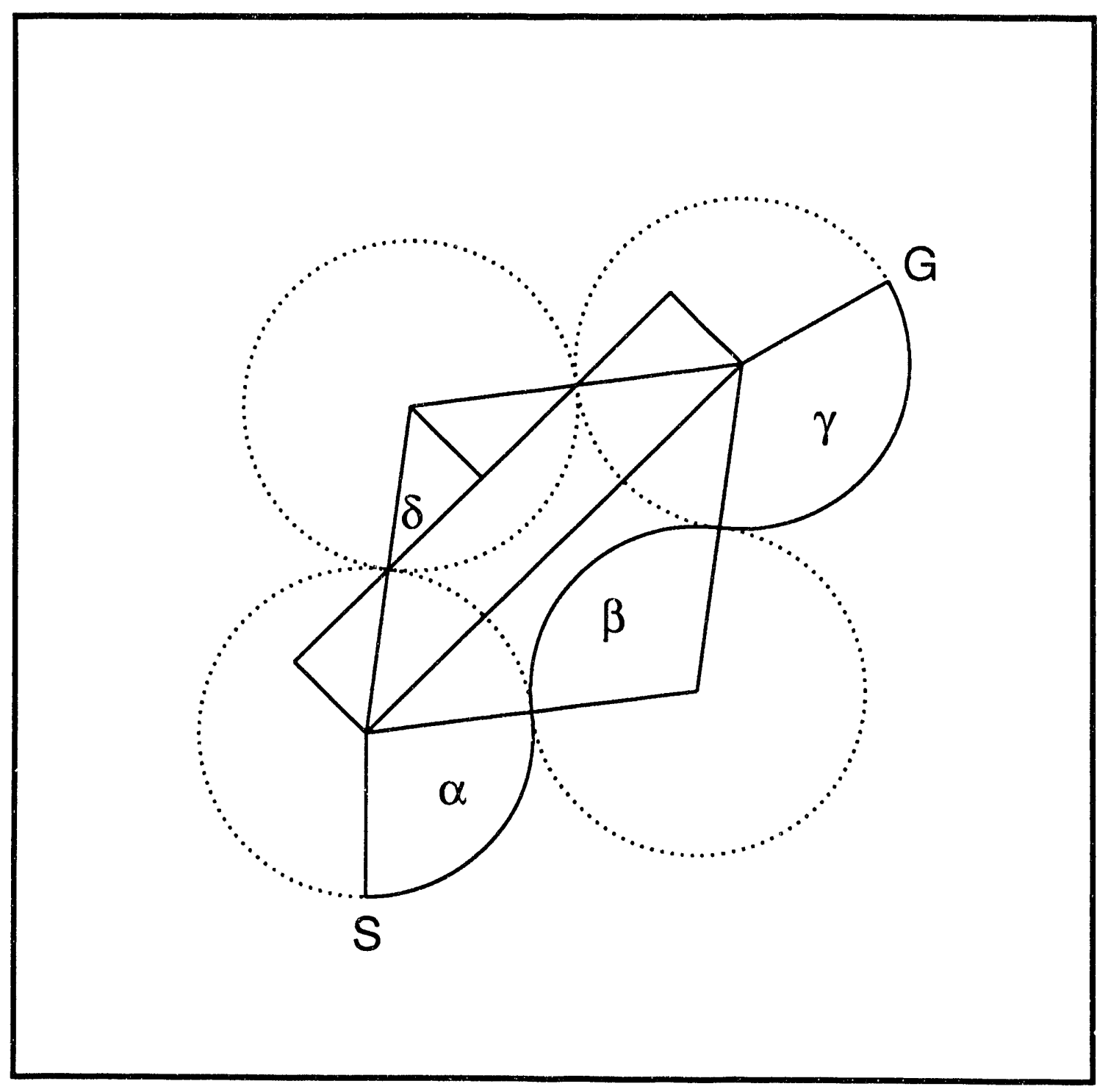

Fig. 15. Calculation of the length of a three circle path. 
ORNL/TM-12289

\section{INTERNAL DISTRIBUTION}

1. V. Alexiades

2. B. R. Appleton

3. J. E. Baker

4. A. L. Bangs

5. M. Beckerman

6. J. Donato

7. J. B. Drake

8. C. W. Glover

9-10. L. J. Gray

11. J. P. Jones

12. H. E. Knee

13. W. F. Lawkins

14-18. S. M. Lenhart

19-23. R. C. Mann

24. E. M. Oblow

25-29. F. G. Pin
30. V. Protopopescu

31-35. D. B. Reister

36. R. F. Sincovec

37. F. J. Sweeney

38. E. C. Uberbacher

39. M. A. Unseren

40-44. R. C. Ward

45. EPMD Reports Office

46-47. Laboratory Records

Department

48. Laboratory Records, ORNL-RC

49. Document Reference Section

50. Central Research Library

51. ORNL Patent Section

\section{EXTERNAL DISTRIBUTION}

52. Professor David Adams, University of Kentucky, Mathematics Department, Lexington, KY 40506

53. Dr. Peter Allen, Department of Computer Science, 450 Computer Science, Columbia University, New York, NY 10027

54. Mr. Harry Alter, Division of Advanced Technology Development, U.S. Department of Energy, Washington, DC 20545

55. Dr. John Baillieul, Aerospace and Mechanical Engineering Department, Boston University, 110 Cummington St., Boston, MA 02215

56. Professor J. D. Birdwell, University of Tennessee, Electrical Engineering Department, Knoxville, TN 37996

57. Dr. Wayne Book, Department of Mechanical Engineering, J. S. Coon Building, Room 306, Georgia Institute of Technology, Atlanta, GA 30332

58. Dr. Johann Borenstein, The University of Michigan, 1101 Beal Ave., Ann Arbor, MI 48109-2110

59. Professor Roger W. Brockett, Wang Professor of Electrical Engineering and Computer Science, Division of Applied Sciences, Harvard University, Cambridge, MA 02138

60. Professor Carl Crane, 202 Nuclear Science Center, University of Florida, Gainesville, FL 32611

61. Dr. Jerry Donato, University of Minnesota, IMA, 206 Church St., SE, Minneapolis, MN 55455

62. Professor John J. Dorning, Department of Nuclear Engineering and Physics, Thornton Hall, McCormick Rd., University of Virginia, Charlottesville, VA 22901

63. Dr. Steven Dubowsky, Massachusetts Institute of Technology, Building 3, Room 469A, 77 Massachusetts Ave., Cambridge, MA 02139

64. Professor L. C. Evans, University of California, Mathematics Department, Berkeley, CA 94720 
65. Professor Ben Fitzpatrick, North Carolina State University, Mathematics Department, Raleigh, NC 27695

66. Dr. Ralph C. Gonzalez, Department of Electrical and Computer Engineering, The University of Tennessee, Knoxville, TN 37996-2100

67. Professor Arthur Heinricher, Worcester Polytechnic Institute, Department of Mathematical Sciences, Worcester, MA 01609

68. Dr. Avi Kak, Department of Electrical Engineering, Purdue University, Northwestern Ave., Engineering Mall, Lafayette, IN 47907

69. Professor Takeo Kanade, Computer Science and Robotics, Carnegie Mellon University, Pittsburgh, PA 15213-3890

70. Dr. James E. Leiss, Route 2, Box 142C, Broadway, VA 22815

71. Dr. Oscar P. Manley, Division of Engineering, Mathematical, and Geosciences, Office of Basic Energy Sciences, ER-15, U.S. Department of Energy - Germantown, Washington, DC 20545

72. Professor Neville Moray, Department of Mechanical and Industrial Engineering, University of Illinois, 1206 West Green St., Urbana, IL 61801

73. Professor James Reneke, Clemson University, Department of Mathematical Sciences, Clemson, SC 29634

74. Dr. Shankar Shastry, Department of EECS, 261-M Cory Hall, University of California, Berkeley, CA 94720

75. Dr. Wes Snyder, Department of Radiology, Bowman Gray School of Medicine, 300 S. Hawthorne Dr., Winston-Salem, NC 27103

76. Professor Hector Sussman, Rutgers University, Mathematics Department, New Brunswick, NJ 08903

77. Professor Delbert Tesar, Department of Mechanical Engineering, 26 San Jacinto, University of Texas, Austin, TX 78712

78. Professor M. A. Trivedi, Department of Electrical and Computer Engineering, The University of Tennessee, Knoxville, TN 37996-2100

79. Professor James S. Tulenko, 202 Nuclear Science Center, University of Florida, Gainesville, FL 32611.

80. Professor David K. Wehe, Department of Nuclear Engineering, 2355 Bonisteel Blvd., The University of Michigan, Ann Arbor, MI 48109-2106

81. Professor Mary F. Wheeler, Department of Mathematical Sciences, Rice University, P.O. Box 1892, Houston, TX 77251

82. Office of Assistant Manager for Energy Research and Development, U.S. Department of Energy, Oak Ridge Operations Office, P.O. Box 2001, Oak Ridge, TN 37831-8600

83-92. Office of Scientific Technical Information, P.O. Box 62, Oak Ridge, TN 37831 
DATE

FILMED

2122193 
